\title{
Adenoid Cystic Carcinoma of Nasal Septum: Report of Two Cases
}

\author{
${ }^{1}$ Priya SR, ${ }^{2}$ Devendra A Chaukar, ${ }^{3}$ Anil KD' Cruz \\ ${ }^{1}$ Fellow, Department of Head and Neck Surgery, Tata Memorial Hospital, Mumbai, Maharashtra, India \\ ${ }^{2}$ Associate Professor, Department of Head and Neck Surgery, Tata Memorial Hospital, Mumbai, Maharashtra, India \\ ${ }^{3}$ Professor, Department of Head and Neck Surgery, Tata Memorial Hospital, Mumbai, Maharashtra, India
}

Correspondence: Priya SR, Fellow, Department of Head and Neck Surgery, Tata Memorial Hospital, Mumbai, Maharashtra India, e-mail: essarpriya@yahoo.com

\begin{abstract}
We present two cases of adenoid cystic carcinoma arising from the nasal septum. The first patient was previously untreated. The second patient had been operated outside with a presumed diagnosis of a benign lesion. We excised both the lesions with wide margins as is advisable for this histology. The histopathology examination in the first case showed adenoid cystic carcinoma with no high-risk features. In the second case, the histopathology revealed no residual malignancy. Adjuvant treatment was, therefore, not given to either patient. The postoperative appearance in both cases was good. They have been disease free on follow-up.
\end{abstract}

Keywords: Adenoid cystic carcinoma, Nasal septum.

\section{INTRODUCTION}

A denoid cystic carcinoma (ADCC) arising from the nasal septum is rare and only a few reports are available in literature. The nasal cavity and the paranasal sinuses are seen to have a worse prognosis than other head and neck sites; however, the nasal cavity tumors have been found to have a better outcome than sites in the paranasal sinuses. Surgery with adequate and clear margins followed by adjuvant radiotherapy when indicated, is the best treatment modality for adenoid cystic cancers. We report two such cases that we encountered at our hospital.

\section{CASE REPORTS}

\section{Case 1}

A 50-year-old man presented with a 6 months history of a left-sided nasal mass. There was history of occasional bloodstained discharge. A nterior rhinoscopy revealed a smooth, nonulcerative mass arising from the cartilaginous nasal septum, the floor and the lateral wall-being free. There was no pal pable cervical lymphadenopathy. A punch biopsy was reported as ADCC. A CT scan (Fig. 1A) confirmed that the bony septum and the paranasal sinuses were clear and that there were no lymph node metastasis. The chest $X$-ray was normal. The tumor was excised by lateral rhinotomy approach. The columella was divided by a horizontal incision. The tumor was seen to be arising from the cartilaginous septum. The entire cartilaginous septum was removed along with a portion of the bony septum. The junction of the lateral alar cartilages and the septum was partly excised for margins.

Grossly the lesion was $1.5 \times 1.5 \times 1 \mathrm{~cm}$ in dimensions. Histology was adenoid cystic carcinoma of a predominantly cribriform pattern. There was no invasion of the underlying cartilage. There was absence of perineural invasion.

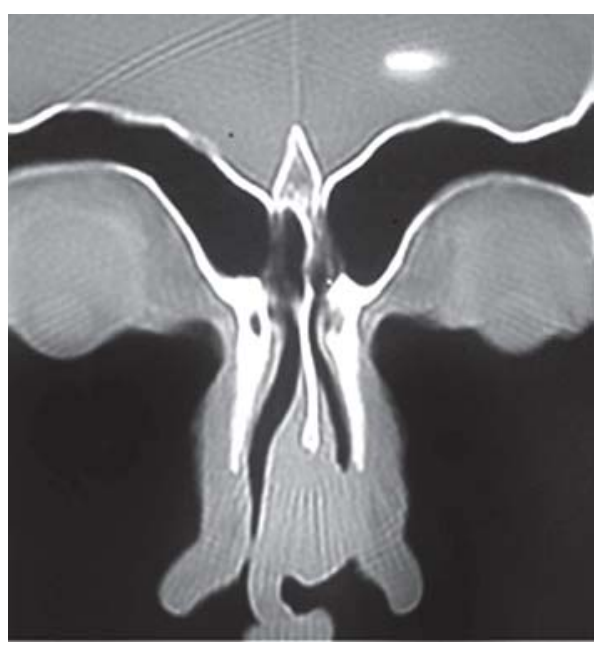

Fig. 1A: CT showing lesion on anterior nasal septum

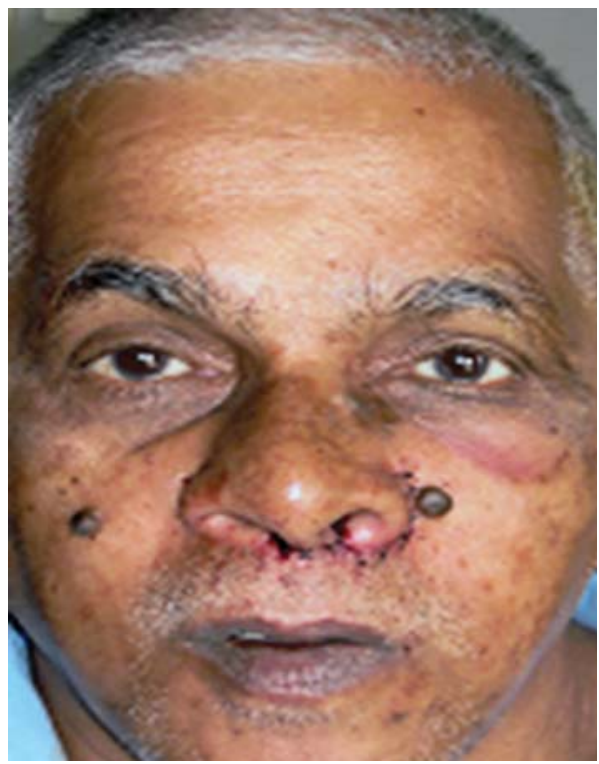

Fig. 1B: Postoperative picture showing minimal deformity 


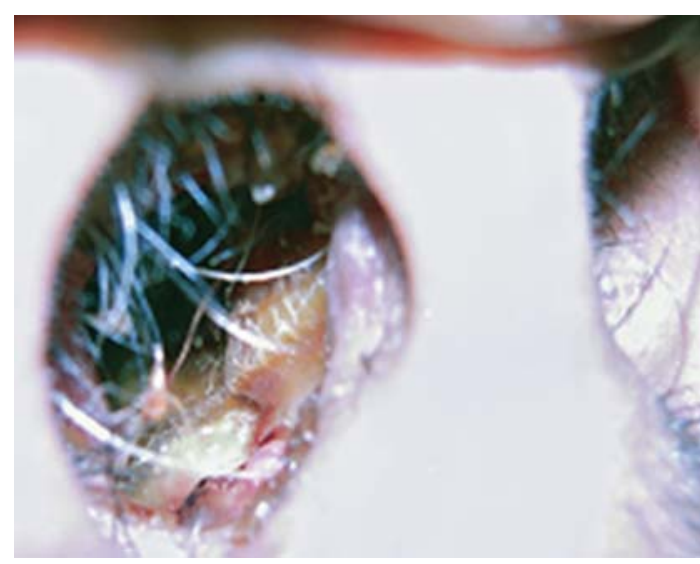

Fig. 2A: Septum showing doubtful lesion anteriorly

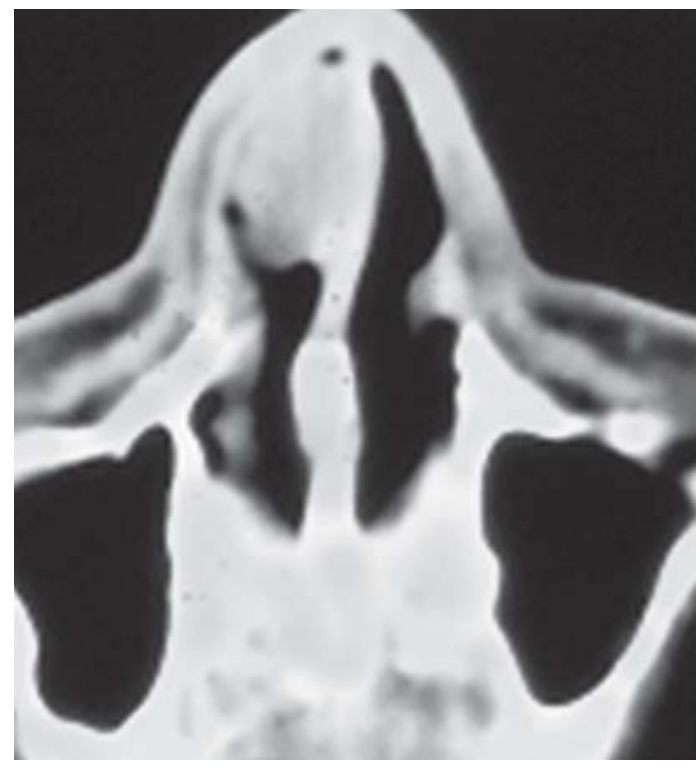

Fig. 2B: CT showing thickened anterior septum

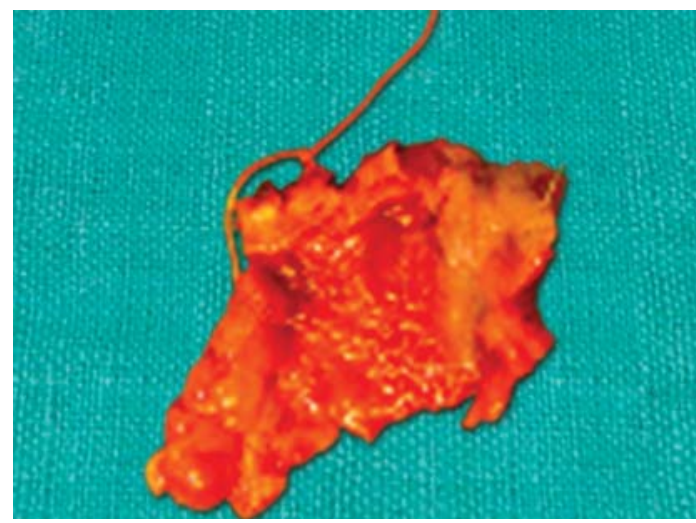

Fig. 2C: Specimen showing lesion in the middle of cartilaginous septum

The postoperative period was uneventful (Fig. 1B). Crusting was the only significant complaint, which was managed by douching. Since it was a low-grade, low-staged tumor, no adjuvant treatment was given.

\section{Case 2}

A 53-year-old man presented with a history of having been operated upon 2 months before, for a right nasal septal mass of 3 years duration. The histopathology of the mass was found to be adenoid cystic carcinoma, and he attended our hospital for further evaluation. On examination, the right side of his nasal septum showed postoperative changes, with a doubtful residual growth (Fig. 2A). A review of the slides from the previous surgery was done, and the histology was confirmed to be ADCC. A computed tomography (Fig. 2B) revealed a thickened anterior nasal septum, the rest of the septum and the lateral wall-being normal. Keeping in view that the previous excision had been with a presumption of a benign lesion, revision surgery was decided upon. Excision was done of the cartilaginous septum along with a cuff of bony septum (Fig. 2C). The specimen showed only inflammatory changes on histopathological examination, with no residual malignancy. Consequently, no adjuvant treatment was given. The early postoperative period was uneventful and the appearance was satisfactory.

B oth above patients have been disease free for 15 months till the time of writing this paper.

\section{DISCUSSION}

ADCC is the second most common tumor of the nasal cavity and paranasal sinuses after squamous cell carcinoma. Sinonasal ADCC accounts for 10 to $25 \%$ of all head and neck A DCC s. ${ }^{1}$ The nasal cavity is the second most common site after the maxillary sinus ${ }^{2}$ for this tumor. However, A DCC limited to the nasal septum has been reported only rarely; the number of patients seen being given as si $x^{3}$ and three ${ }^{4}$ in the literature.

ADCC can be classified histologically into cribriform, tubular and solid types. The cribriform is the commonest and has the best outcome, while the solid tumors are least common and have the worst prognosis. ${ }^{5}$ Patients with nasal cavity disease had better overall and disease-specific survival as compared with tumors in other sites in the PNS. ${ }^{6}$ The clinical stage, particularly the T stage, is the critical factor in deciding the outcome of salivary gland cancer as per data collected at M emorial Sloane K ettering Center. ${ }^{7}$ Other prognostic factors are size of tumor, margins of resection, lymph node metastasis, perineural invasion or invasion of bone vasculature muscle or extraglandular tissue. Salivary gland tumors of T1 and T2 stage, i.e. less than $4 \mathrm{~cm}$ in size, do well irrespective of histological grade. Radiotherapy is beneficial for tumors over this size, having little impact over smaller tumors. ${ }^{8}$

The recommended treatment of sinonasal ADCC is complete surgical resection with adequate and tumor-free margins, followed by radiotherapy. Radiation is not curative, but in the postoperative setting has been shown to be effective in improving local control. Patients who undergo surgery with postoperative radiotherapy as the primary 
treatment modality have significantly improved overall as well as disease-specific survival, as compared with those being treated with other modalities including surgery alone and radiation alone. ${ }^{5}$ Aggressive therapy for the primary tumor does lead to a high and long-term local control rate, though it does not influence survival, because of metastatic disease. $^{8}$

ADCCS typically recur locally, the rate of recurrence being $100 \%$ for solid tumors and $59-89 \%$ for lower grade tumors. ${ }^{9} \mathrm{~A}$ recent study found an overall recurrence rate of $65 \%$ in sinonasal A DCC, despite surgery and radiotherapy. ${ }^{6}$ These tumors metastasize frequently (35 to $50 \%$ ), usually to lung and bone. ${ }^{10}$ The 5 -year survival rates for low-stage, low-grade tumors are roughly $85 \%$, but at 10 years, all grades do equally, with overall survival of less than $50 \% .{ }^{11}$

B oth patients in this report presented early. The tumor was present in a relatively easily accessible area of the cartilaginous nasal septum. Surgically clear margins were achieved without significantly disturbed cosmesis. The pathology of the tumor in the first patient was favorable viz a T1 lesion, cribriform pattern, absence of perineural invasion, clear margins. Hence, no further adjuvant treatment in the form of radiotherapy was found to be warranted. Surgery in the second case was to ensure wide margins of residual malignancy, if any. Secondary reconstruction for nasal dorsum saddling will be planned, if so required, at a later stage.

\section{REFERENCES}

1. Rhee CS, W on TB, Lee CH, et al. A denoid cystic carcinoma of the sinonasal tract: Treatment results. Laryngoscope 2006;116:982-86.

2. Dulguerev $P$, J acobsen M S, A llal AS, et al. Nasal and paranasal sinus carcinoma: A re we making progress? A series of 220 patients and a systematic review. Cancer 2001;92:3012-29.

3. Handa Y, Y amamoto H, Y amakawa J, Hayashi T, Rita Y. A case report of adenoid cystic carcinoma of the nasal septum. Nippon Jibiinkoka Gakkai Kaiho 1992;95:505-09.

4. Fleury P, B asset J M, Compere J F, Pansier P. Rare tumors of the nasal septum. Eight reported cases. A nnal of Otolaryngol Chir Cervicofacial 1979;96:767-79.

5. Wiseman SM, Popat SR, Rigual NR, et al. A denoid cystic carcinoma of the paranasal sinuses or nasal cavity: A 40-year review of 35 cases. Ear Nose Throat J ournal 2002;81:510-17.

6. Lupinetti Allison D, Roberts Dianna B, Williams Michelle D. Sinonasal adenoid cystic carcinoma. The M D A nderson Cancer Center Experience. Cancer 2007;110:2726-31.

7. Speight PM , Barrett A W. Prognostic factors in malignant tumors of the salivary glands. B ritish J ournal of $O$ ral and M axillofacial Surgery 2009;D OI:10.1016/j.bjoms.2009.03.017.

8. Renchan AG, Gleave EN, Slevin NJ, M cGurk M. Clinicopathological and treatment related factors influencing survival in parotid cancer. B ritish J Cancer 1999;80:1296-1300.

9. Perzin KH, Gullane P, ClairmontA C. A denoid cystic carcinomas arising in salivary glands: A correlation of histologic features and clinical course. Cancer 1978;42:265-82.

10. Spiro RH. Distant metastasis in adenoid cystic carcinoma of salivary gland origin. American Journal of Surgery 1997;174:495-98.

11. Spiro RH. Distant metastasis in adenoid cystic carcinoma. Is this cancer curable and where does it fail? In: M cGurk M, Renchan A (Eds). Controversies in management of salivary gland disease. Oxford: Oxford U niversity Press 2001;207-11. 